\title{
Effect of Motor Imagery on the F-Wave Parameters in Hemiparetic Stroke Survivors
}

\author{
Mahshid Naseri, $\mathrm{MD}^{1}$, Peyman Petramfar, $\mathrm{MD}^{2}$, Alireza Ashraf, $\mathrm{MD}^{1}$ \\ ${ }^{1}$ Department of Physical Medicine and Rehabilitation and Shiraz Burn Research Centre, \\ Shiraz University of Medical Sciences, Shiraz; ${ }^{2}$ Department of Neurology, \\ Shiraz University of Medical Shiraz, Iran
}

Objective To assess the effect of motor imagery, as a rehabilitation method in stroke, on F-wave parameters that undergo changes during upper motor neuron involvement.

Methods Twenty-one fully conscious hemiparetic stroke survivors with a completely plegic hand (power 0/5) and a minimum interval of 72 hours since stroke were recruited into this study. The mean F-wave latency, amplitude, and persistence in the median and ulnar nerves were measured in both the affected and non-affected sides at rest and in the paretic hand during a mental task. Comparison was made between data from the affected hand and the non-affected hand as well as between data from the affected hand at baseline and during motor imagery.

Results Patients had significantly different F-wave persistence between the affected and non-affected sides (paired $\mathrm{t}$-test, $\mathrm{p}<0.001$ ). Motor imagery could improve F-wave persistence in both the investigated nerves (paired t-test, $\mathrm{p}=0.01$ for ulnar nerve and $\mathrm{p}<0.001$ for median nerve) and F-response amplitude in the median nerve (paired $\mathrm{t}$-test, $\mathrm{p}=0.01$ ) of the affected limb.

Conclusion The amplitude and persistence of F-wave were improved during motor imagery, representing F-wave facilitation. This result suggests that motor imagery can restore motor neuron excitability, which is depressed after stroke.

Keywords Stroke, Hemiplegia, Motor imagery, F-wave

Received April 3, 2014; Accepted June 9, 2014

Corresponding author: Alireza Ashraf

Department of Physical Medicine and Rehabilitation and Shiraz Burn Research Centre, Shiraz University of Medical Sciences, Shiraz 7134844119, Iran

Tel: +98-7112319040, Fax: +98-7112319040, E-mail: md.pmr90@gmail. com

(c) This is an open-access article distributed under the terms of the Creative Commons Attribution Non-Commercial License (http://creativecommons. org/licenses/by-nc/4.0) which permits unrestricted noncommercial use, distribution, and reproduction in any medium, provided the original work is properly cited.

Copyright $\odot 2015$ by Korean Academy of Rehabilitation Medicine

\section{INTRODUCTION}

Stroke is a major cause of morbidity. Many subjects remain permanently disabled, despite intensive rehabilitation. After management of the acute phase, the patients should participate in exercise programs as soon as possible to activate the process of recovery and neural re-organization [1]. Motor imagery is a process during which a specific action is reproduced mentally without any real movements, and the same neural and muscular structures are activated during physical activity [1,2]. Many studies suggested that a stroke rehabilitative regimen 
augmented by mental practice renders a greater impact on upper extremity movement, gait, and other functional tasks than physical therapy only [3-13]. A recent systematic review concluded that mental practice in conjunction with conventional physical therapy can not only be effective for functional rehabilitation of both upper and lower extremities, but also for the recovery of daily activities and skills [14].

F-waves-produced by backfiring of the alpha motor neurons-have been used as a probe for motor cortex excitability and F-wave activity can be modified by motor imagery based on some previous reports [15]. In 2008, Taniguchi et al. [16] observed a decline in F-wave persistence and amplitude after relaxation task which recovered quickly after exercise and changed little with imagery task. They stated that motor imagery without actual muscle contraction can effectively counter the suppression induced by relaxation task and maintain the excitability of the anterior horn cells. Therefore, this maneuver may restore the spinal excitability even while testing a paretic muscle and this result was reproduced later by Hara et al. [17]. To elucidate the physiologic mechanisms underlying motor imagery, Fujisawa et al. [18], conducted a study in 2011 and proposed that mental simulation counters rest-induced suppression primarily by restoring spinal excitability.

In two studies performed by Milanov $[19,20]$, the Fwave was found to be increased in amplitude, duration, latency, and persistence on the spastic side of spastic hemiparetic patients. Voluntary muscle contraction enlarged the F-wave amplitude in normal subjects and patients with lower motor neuron disorders, but this facilitation was not seen in patients with upper motor neuron disorder. Differences between the upper motor neuron disorder group and the normal and lower motor neuron disorder groups with respect to the F-wave amplitudes at rest and during voluntary contraction was the basis for the theory that F-waves can be used as a monitor of upper motor neuron disorder [21].

To the best of our knowledge, this is the first study to investigate the effect of motor imagery on the F-wave elicited from a paretic muscle as a result of stroke in contrast to the prior studies that evaluated F-responses in intentionally inactivated normal muscles. We aimed to assess whether the F-wave parameters in the paretic side of stroke patients change during motor imagery. The hypothesis is that in a paretic muscle following stroke, mental practice might improve the F-wave parameters which can be due to restoration of the spinal excitability via corticospinal facilitation. It means that in clinical practice, motor imagery might be able to induce some neuroplastic changes in the brain of stroke survivors that lead to motor recovery. This effect can be one of the mechanisms involved in stroke neurorehabilitation by performing mental tasks.

\section{MATERIALS AND METHODS}

Fully conscious hemiparetic stroke survivors (Glasgow Coma Scale 15/15) between 25 and 65 years of age, in whom the diagnosis was made based on the physical examination and brain imaging findings, with distal upper extremity power of $0 / 5$ based on manual muscle testing and minimum interval of 72 hours since stroke were recruited into this study. Patients in a stable neurologic condition, who were referred to our university electrodiagnostic clinic between February 2012 and January 2013, were enrolled in this study. Subjects with decreased level of consciousness, those suffering from Wernicke's or global aphasia or asomatognosia, and patients with a past history of peripheral neuropathy or cervical radiculopathy were excluded. Patients were examined after they gave their informed consent. All patients underwent detailed upper limb nerve conduction studies and those with electrophysiological findings suggestive of any peripheral nerve involvement were also excluded. Research protocol was approved by our university ethics committee, and the study was performed in accordance with the ethical standards laid down in the 1964 Declaration of Helsinki and its later amendments.

It is supposed that patients with spasticity have some differences in the F-wave parameters, such as amplitude and persistence, compared to the subjects with normal or flaccid muscle tone. To investigate whether motor imagery has a different effect in stroke patients who have a different muscle tone, we assessed the muscle tone in the elbow region of the paretic side in patients according to the Ashworth scale and enrolled the patients who received a score of zero in the flaccid category. Other patients with a score equal to or more than one were enrolled in the spastic group.

The electrodiagnostic tests were performed by using a 
Medelec Synergy electromyography instrument (VIASYS HealthCare, Surrey, UK) with bar electrodes as stimulators and recorders. We tried to maintain the skin temperature at least $31^{\circ} \mathrm{C}$ for recording the $\mathrm{F}$-wave parameters in rooms with similar constant temperatures between $23^{\circ} \mathrm{C}$ and $25^{\circ} \mathrm{C}$. F-waves were elicited by supramaximal electrical shock delivery to either the median nerve or the ulnar nerve of each hand at the wrist, $8 \mathrm{~cm}$ proximal to the recording sites at the abductor pollicis brevis (APB) or abductor digiti minimi (ADM) muscle bellies. These two nerves are accessible motor nerves that are usually assessed in conventional upper limb electrodiagnostic studies. We recorded the amplitude, latency, and persistence of F-waves in both hands at rest and in the paretic hand during motor imagery task. For every trial, we delivered 10 stimuli at a frequency of $1 \mathrm{~Hz}$ and measured the mean latency and average peak-to-peak amplitude of all detectable responses. Also, we recorded the total number of detectable responses after 10 stimuli as the persistence. During each trial, we tried to ensure that the patient did not make any unintentional hand movements.

The electrodiagnostic parameters employed in our study were as follows [22]: sweep speed $5 \mathrm{~ms} /$ div; sensitivity $100-200 \mu \mathrm{V} /$ div; and band pass filter 10-10,000 Hz. The maximal stimulus was determined by increasing the intensity or pulse duration to the point at which the largest compound muscle action potentials could be recorded. Supramaximal stimulus for eliciting the F-wave was considered to be $20 \%$ higher than the maximal stimulus.

Motor imagery task consisted of abduction of the little finger for the ulnar nerve and thumb opposition for the median nerve. First, we taught the finger movements to the patients and supervised them while they performed the task using the non-affected side. Then, we asked the patients to perform the imaginary tasks for the affected limbs. Motor imagery was conducted when the patient declared that he/she had understood the process and was ready for performing the task during the study. Subsequently, F-wave recording was performed during motor imagery.

Data were analyzed using SPSS ver. 17 (SPSS Inc., Chicago, IL, USA). Comparison was made between the Fwave parameters recorded from the paretic hand and the non-paretic hand and also between F-response parameters recorded at rest and during the motor imagery task

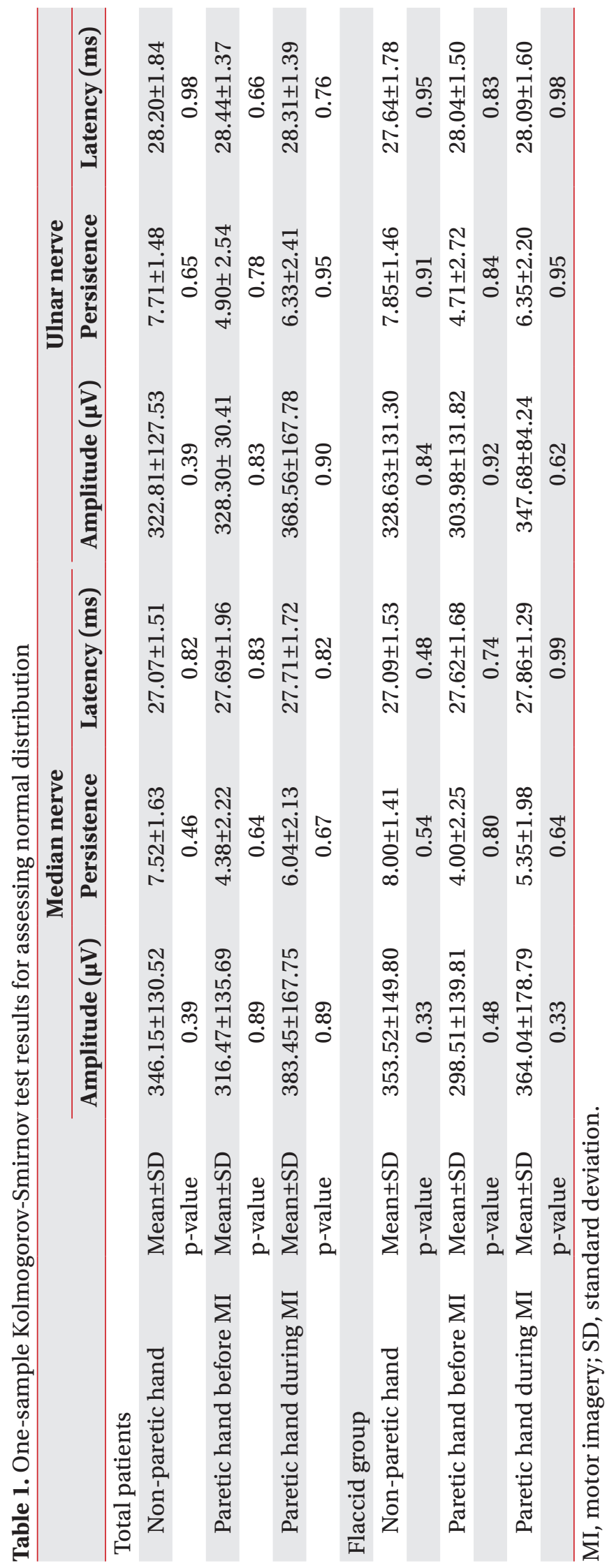


from the paretic side only. According to the inclusion criteria, we recruited the patients with a completely plegic hand (muscle power of $0 / 5$ ), and therefore, when the patients performed mental practice they could not unintentionally contract the muscle that was being investigated. This helped us to isolate the effect of motor imagery on the F-wave parameters. The F-wave changes during motor imagery on the unaffected side might be contaminated by the effect of voluntary contraction. We know that slight contraction of the muscle under investigation can facilitate observation of F-waves. Therefore, we did not make a comparison between the F-waves parameters at baseline and during motor imagery in the unaffected side [23]. We used one-sample Kolmogorov-Smirnov test for checking normality of the data distribution. Paired t-test was used for analysis of normally distributed data and Wilcoxon signed-rank test was used for data that did not have normal distribution. The $\mathrm{p}$-values less than 0.05 were considered statistically significant.

\section{RESULTS}

Among the 21 patients recruited in our study, $42.9 \%$ were females and $57.1 \%$ were males. Patients had mean age of $48.61 \pm 9.99$ years. The time interval since the onset of stroke to the day of the study was variable, and it ranged from 4 to 75 days with a mean of $32.66 \pm 20.56$ days.
The mean time spent after stroke was $52.14 \pm 20.51$ days in patients with spastic paresis compared to $22.92 \pm 12.24$ days in patients suffering from flaccid paresis. With respect to the tone, 14 patients $(66.7 \%)$ had upper limb flaccid paresis compared to 7 patients (33.3\%) who had spastic paresis based on the physical examination.

According to the one-sample Kolmogorov-Smirnov test results, the overall data obtained from the flaccid group were normally distributed (Table 1). Due to normal data distribution, we used the paired t-test and found an overall significant difference in F-wave persistence for both the median and ulnar nerves between paretic and non-paretic hands (Table 2). During the motor imagery task, there was a significant change in the median nerve F-wave amplitude and persistence compared to the baseline. Also, we observed a statistically significant improvement in the ulnar nerve F-wave persistence, but a non-significant improvement in the ulnar nerve F-wave amplitude (Table 3).

In the group with flaccid paresis, the results were similar to the total patients' analyses and there was a significant improvement in the median nerve F-wave amplitude and the median and ulnar nerve F-wave persistence during motor imagery. Although the F-wave amplitude of the ulnar nerve was also improved, this result did not reach statistical significance. In this group, a statistically significant difference was seen only in the F-wave per-

Table 2. Comparison of median and ulnar nerve F-wave parameters between paretic and non-paretic hands of patients before motor imagery

\begin{tabular}{lccccccc}
\hline & \multicolumn{3}{c}{ Median nerve } & \multicolumn{3}{c}{ Ulnar nerve } \\
\cline { 2 - 4 } \cline { 7 - 8 } & Amplitude $(\boldsymbol{\mu V})$ & Persistence & Latency $(\mathbf{m s})$ & & Amplitude $(\boldsymbol{\mu V})$ & Persistence & Latency (ms) \\
\hline Non-paretic hand & $346.15 \pm 130.52$ & $7.52 \pm 1.63$ & $27.07 \pm 1.51$ & & $322.81 \pm 127.53$ & $7.71 \pm 1.48$ & $28.20 \pm 1.84$ \\
Paretic hand & $316.47 \pm 135.69$ & $4.38 \pm 2.22$ & $27.69 \pm 1.96$ & & $328.30 \pm 30.41$ & $4.90 \pm 2.54$ & $28.44 \pm 1.37$ \\
p-value & 0.21 & $<0.001$ & 0.09 & 0.76 & $<0.001$ & 0.36 \\
95\% CI & -18.28 to 77.63 & 1.99 to 4.28 & -1.36 to 0.12 & -43.87 to 32.90 & 1.84 to 3.77 & -0.78 to 0.30 \\
\hline
\end{tabular}

Table 3. Comparison of median and ulnar nerve F-wave parameters at rest and during motor imagery in paretic hands

\begin{tabular}{lccccccc}
\hline & \multicolumn{3}{c}{ Median nerve } & \multicolumn{3}{c}{ Ulnar nerve } \\
\cline { 2 - 4 } \cline { 6 - 7 } & Amplitude $(\boldsymbol{\mu V})$ & Persistence & Latency $(\mathbf{m s})$ & & Amplitude $(\boldsymbol{\mu V})$ & Persistence & Latency (ms) \\
\hline Paretic hand before MI & $316.47 \pm 135.69$ & $4.38 \pm 2.22$ & $27.69 \pm 1.96$ & & $328.30 \pm 30.41$ & $4.90 \pm 2.54$ & $28.44 \pm 1.37$ \\
Paretic hand during MI & $383.45 \pm 167.75$ & $6.04 \pm 2.13$ & $27.71 \pm 1.72$ & & $368.56 \pm 167.78$ & $6.33 \pm 2.41$ & $28.31 \pm 1.39$ \\
p-value & 0.01 & $<0.001$ & 0.94 & 0.15 & 0.01 & 0.68 \\
95\% CI & -115.75 to -18.20 & -2.43 to -0.90 & -0.81 to 0.76 & -97.41 to 16.89 & -2.47 to -0.38 & -0.54 to 0.80 \\
\hline
\end{tabular}

MI, motor imagery. 
Table 4. Comparison of median and ulnar nerve F-wave parameters between paretic and non-paretic hands of patients according to the upper limb tone before motor imagery

\begin{tabular}{|c|c|c|c|c|c|c|}
\hline & \multicolumn{3}{|c|}{ Median nerve } & \multicolumn{3}{|c|}{ Ulnar nerve } \\
\hline & Amplitude $(\mu \mathrm{V})$ & Persistence & Latency (ms) & Amplitude $(\mu \mathrm{V})$ & Persistence & Latency (ms) \\
\hline \multicolumn{7}{|l|}{ Flaccid group } \\
\hline Non-paretic hand & $353.52 \pm 149.80$ & $8.00 \pm 1.41$ & $27.09 \pm 1.53$ & $328.63 \pm 131.30$ & $7.85 \pm 1.46$ & $27.64 \pm 1.78$ \\
\hline Paretic hand & $298.51 \pm 139.81$ & $4.00 \pm 2.25$ & $27.62 \pm 1.68$ & $303.98 \pm 131.82$ & $4.71 \pm 2.72$ & $28.04 \pm 1.50$ \\
\hline $\mathrm{p}$-value & 0.07 & $<0.001$ & 0.26 & 0.25 & $<0.001$ & 0.14 \\
\hline $95 \% \mathrm{CI}$ & -6.83 to 116.86 & 2.53 to 5.46 & -1.49 to 0.44 & -20.34 to 69.64 & 1.88 to 4.40 & -0.97 to 0.15 \\
\hline \multicolumn{7}{|l|}{ Spastic group } \\
\hline Non-paretic hand & $331.40 \pm 88.21$ & $6.57 \pm 1.71$ & $27.02 \pm 1.57$ & $311.18 \pm 128.94$ & $7.42 \pm 1.61$ & $29.33 \pm 1.50$ \\
\hline Paretic hand & $352.40 \pm 129.48$ & $5.14 \pm 2.11$ & $27.82 \pm 2.58$ & $376.92 \pm 122.08$ & $5.28 \pm 2.28$ & $29.24 \pm 0.54$ \\
\hline $\mathrm{p}$-value* & 0.39 & 0.04 & 0.31 & 0.04 & 0.02 & 0.86 \\
\hline
\end{tabular}

*Wilcoxon signed-rank test.

Table 5. Comparison of median and ulnar nerve F-wave parameters at rest and during motor imagery in paretic hands according to the upper limb tone

\begin{tabular}{|c|c|c|c|c|c|c|}
\hline & \multicolumn{3}{|c|}{ Median nerve } & \multicolumn{3}{|c|}{ Ulnar nerve } \\
\hline & Amplitude $(\mu \mathrm{V})$ & Persistence & Latency (ms) & Amplitude $(\mu \mathrm{V})$ & Persistence & Latency (ms) \\
\hline \multicolumn{7}{|l|}{ Flaccid group } \\
\hline Paretic hand before MI & $298.51 \pm 139.81$ & $4.00 \pm 2.25$ & $27.62 \pm 1.68$ & $303.98 \pm 131.82$ & $4.71 \pm 2.72$ & $28.04 \pm 1.50$ \\
\hline Paretic hand during MI & $364.04 \pm 178.79$ & $5.35 \pm 1.98$ & $27.86 \pm 1.29$ & $347.68 \pm 84.24$ & $6.35 \pm 2.20$ & $28.09 \pm 1.60$ \\
\hline $\mathrm{p}$-value & 0.04 & 0.01 & 0.59 & 0.30 & 0.04 & 0.91 \\
\hline $95 \%$ CI & -127.45 to -3.59 & -2.33 to -0.38 & -1.21 to 0.72 & -131.90 to 44.51 & -3.23 to -0.04 & -0.98 to 0.88 \\
\hline \multicolumn{7}{|l|}{ Spastic group } \\
\hline Paretic hand before MI & $352.40 \pm 129.48$ & $5.14 \pm 2.11$ & $27.82 \pm 2.58$ & $376.92 \pm 122.08$ & $5.28 \pm 2.28$ & $29.24 \pm 0.54$ \\
\hline Paretic hand during MI & $422.28 \pm 147.99$ & $7.42 \pm 1.81$ & $27.42 \pm 2.46$ & $410.31 \pm 131.29$ & $6.28 \pm 2.98$ & $28.74 \pm 0.76$ \\
\hline p-value* & 0.02 & 0.02 & 0.79 & 0.04 & 0.03 & 0.17 \\
\hline
\end{tabular}

MI, motor imagery.

*Wilcoxon signed-rank test.

sistence of median and ulnar nerves between both the hands (Tables 4, 5).

Due to the small sample size of the group with spastic upper limb paresis, we used Wilcoxon signed-rank test for the data analysis. In this group, a significant improvement was seen in the median and ulnar nerve F-wave amplitude and persistence during motor imagery. The comparison between normal and paretic hands showed a statistically significantly lower median and ulnar nerve F-wave persistence and higher ulnar nerve F-wave amplitude in paretic hands than in non-paretic hands (Tables 4, $5)$.

\section{DISCUSSION}

Previous studies have provided evidence for the role of motor imagery in rehabilitation of patients with different disorders, such as stroke. Although F-wave has been used as a probe for motor cortex excitability with clinical utility in stroke patients [15], it has never been studied for investigating the underlying mechanism involved in the rehabilitation of these patients by performing mental practice. Our study not only showed a significant difference in some F-wave parameters between the paretic and non-paretic sides of patients, but it also demonstrated a significant improvement in some F-wave parameters of paretic hands during motor imagery. 
A survey by Chroni et al. [23] assessed the changes in ulnar nerve F-wave variables associated with acute hemiparetic stroke, and they could not find any significant difference between F-wave variables in the affected or unaffected side of fully conscious stroke survivors and control subjects. They suggested that any changes in persistence or maximum amplitude during the immediate phase after stroke is due to the decreased level of consciousness. We excluded patients in the acute phase of stroke and recruited only fully conscious patients after a minimum interval of 72 hours since stroke. We found a significantly different F-wave persistence in the affected and nonaffected hands recorded from both the median and ulnar nerves in this study sample.

Similar to our results, some other studies reported changes in the F-wave parameters after stroke that could be observed even earlier than changes in the clinical status $[24,25]$. Differences were observed in the persistence of F-responses in patients with different muscular tones [26] and in an examination of 120 patients with spastic hemiparesis after stroke, the F-wave latencies were prolonged on the affected side [21]. We did not find any significant difference in F-wave latencies between the paretic and non-paretic hands either in the total analysis or after subgroup analysis according to the muscle tone. Interestingly, in contrast to the patients with flaccid hemiparesis in whom the non-affected hands showed greater mean amplitude of the F-wave than the paretic hands, the patients with spastic paresis had greater F-wave mean amplitude on their affected side. The more prolonged duration of paresis in spastic patients might help to explain this finding because reduced spinal motor neuron excitability is reported to be present in the initial stages of stroke, which is followed by increased motor neuron excitability within 90 days [26]. The F-wave was already found to be increased in amplitude, duration, and persistence on the spastic side in patients studied by Milanov [19]. This confirms the concept that F-wave parameters are related to spinal motor neuron excitability, which was previously demonstrated by Drozdowski et al. [25]; however, our study population included a limited number of spastic patients, which did not allow for making a definite conclusion.

The finding of an increase in the amplitude and persistence of the F-wave due to motor imagery observed in this study was previously reported in a pioneering survey investigating the effect of motor imagery on the F-wave elicited in 12 healthy subjects [17]. F-wave amplitude increment due to mental practice has also been observed in another study as the effect of motion imagery to counter the effect of suppression induced by three hours of muscle immobilization [16].

Only F-wave persistence and mean F-wave amplitude to a lesser degree would reflect the change in motor unit excitability due to corticospinal tract and other upper motor neuron influences as stated by Rivner [15]. This might explain why we could not find a statistically significant change in the ulnar nerve F-wave amplitude on the affected sides of patients especially in patients of the flaccid group, during motor imagery; while an obvious significant increase was seen in the ulnar nerve F-wave persistence during mental task. The degree of degeneration of the lateral corticospinal tracts is proposed to be parallel that of fiber loss in ventral roots in stroke patients, and the estimated motor unit number in the moderate to severely affected hemiplegic side decreases after stroke [27]. It seems that either the facilitatory or inhibitory effects of cortical activity are manifested at the spinal level which would be reflected in the F-wave parameters [15]. Therefore, mental practice might provide a facilitatory effect that is needed to counter the reduced anterior horn cell excitability induced by stroke and to consequently improve the F-wave amplitude and persistence elicited from distal muscles.

Similar to this study, the study by Hara et al. [17] showed no significant changes in F-wave latencies after mental practice. This might be due to the theory that the F-wave is mostly generated by large, fast conducting motor units leaving little room for motor imagery to recruit the faster conducting axons. In contrast to these two studies, Taniguchi et al. [16], found a shift toward longer F-wave latencies after imagery task and suggested a greater facilitation of the small, slow conducting motor units as a result of mental practice which is consistent with the size principle. On the other hand, they suggested that motor imagery might be unable to sustain normal reactivity of the anterior horn cells leading to a delay in recurrent discharge [16].

However, there are some limitations to this study. We selected the patients with a completely plegic hand according to the physical examination and we had some difficulties in differentiating between $0 / 5$ and $1 / 5$ power 
in the APB or ADM muscles. If patients had not been selected carefully, the ability to contract the muscle might lead to the generation of F-wave not exclusively due to motor imagery. Although we visually monitored patients' performance, it might not be adequate for confirming the ability to perform motor imagery task without muscle contraction. Lack of an objective parameter for monitoring the real performance of the mental task and its accuracy is another limitation of this study. Finally, it should be noted that in spastic or hypertonic hands, electrical shock may induce flexor synergy and significant changes in the F-wave parameters. We tried to visually monitor the patients' hands during the trials of F-wave recording to ensure that there was no additional hand movement; however, no strict method for wrist and hand fixation was followed.

It seems that motor imagery can improve some F-wave parameters in the plegic muscles of stroke survivors. This improvement might be due to restoration of motor neuron excitability. We recommend conducting further well-designed studies in the near future using more task-specific motor imagery techniques and appropriate monitoring tools, such as surface electromyography, for ensuring that the F-responses are not contaminated by muscle contraction.

\section{CONFLICT OF INTEREST}

No potential conflict of interest relevant to this article was reported.

\section{ACKNOWLEDGMENTS}

This study is a part of the thesis of Dr. Mahshid Naseri (No. 4396). Hence, we would like to thank Shiraz University of Medical Sciences for supporting the research.

\section{REFERENCES}

1. Zimmermann-Schlatter A, Schuster C, Puhan MA, Siekierka E, Steurer J. Efficacy of motor imagery in post-stroke rehabilitation: a systematic review. J Neuroeng Rehabil 2008;5:8.

2. Page SJ, Levine P, Leonard A. Mental practice in chronic stroke: results of a randomized, placebocontrolled trial. Stroke 2007;38:1293-7.
3. Page SJ, Dunning K, Hermann V, Leonard A, Levine P. Longer versus shorter mental practice sessions for affected upper extremity movement after stroke: a randomized controlled trial. Clin Rehabil 2011;25:627-37.

4. Page SJ, Murray C, Hermann V, Levine P. Retention of motor changes in chronic stroke survivors who were administered mental practice. Arch Phys Med Rehabil 2011;92:1741-5.

5. Dettmers C, Benz M, Liepert J, Rockstroh B. Motor imagery in stroke patients, or plegic patients with spinal cord or peripheral diseases. Acta Neurol Scand 2012; 126:238-47.

6. Guttman A, Burstin A, Brown R, Bril S, Dickstein R. Motor imagery practice for improving sit to stand and reaching to grasp in individuals with poststroke hemiparesis. Top Stroke Rehabil 2012;19:306-19.

7. Page SJ, Levine P, Khoury JC. Modified constraint-induced therapy combined with mental practice: thinking through better motor outcomes. Stroke 2009;40: 551-4.

8. Page SJ, Szaflarski JP, Eliassen JC, Pan H, Cramer SC. Cortical plasticity following motor skill learning during mental practice in stroke. Neurorehabil Neural Repair 2009;23:382-8.

9. Malouin F, Richards CL, Durand A, Doyon J. Added value of mental practice combined with a small amount of physical practice on the relearning of rising and sitting post-stroke: a pilot study. J Neurol Phys Ther 2009;33:195-202.

10. Dickstein R, Dunsky A, Marcovitz E. Motor imagery for gait rehabilitation in post-stroke hemiparesis. Phys Ther 2004;84:1167-77.

11. Page SJ, Levine P, Leonard AC. Effects of mental practice on affected limb use and function in chronic stroke. Arch Phys Med Rehabil 2005;86:399-402.

12. Dunsky A, Dickstein R, Ariav C, Deutsch J, Marcovitz E. Motor imagery practice in gait rehabilitation of chronic post-stroke hemiparesis: four case studies. Int J Rehabil Res 2006;29:351-6.

13. Dijkerman HC, Ietswaart M, Johnston M, MacWalter RS. Does motor imagery training improve hand function in chronic stroke patients? A pilot study. Clin Rehabil 2004;18:538-49.

14. Garcia Carrasco D, Aboitiz Cantalapiedra J. Effectiveness of motor imagery or mental practice in functional recovery after stroke: a systematic review. Neuro- 
logia 2013 Apr 16 [Epub]. http://dx.doi.org/10.1016/ j.nrl.2013.02.003.

15. Rivner MH. The use of F-waves as a probe for motor cortex excitability. Clin Neurophysiol 2008;119:12156.

16. Taniguchi S, Kimura J, Yamada T, Ichikawa H, Hara M, Fujisawa R, et al. Effect of motion imagery to counter rest-induced suppression of $\mathrm{F}$-wave as a measure of anterior horn cell excitability. Clin Neurophysiol 2008; 119:1346-52.

17. Hara M, Kimura J, Walker DD, Taniguchi S, Ichikawa H, Fujisawa R, et al. Effect of motor imagery and voluntary muscle contraction on the F wave. Muscle Nerve 2010;42:208-12.

18. Fujisawa R, Kimura J, Taniguchi S, Ichikawa H, Hara M, Shimizu $\mathrm{H}$, et al. Effect of volitional relaxation and motor imagery on F wave and MEP: do these tasks affect excitability of the spinal or cortical motor neurons? Clin Neurophysiol 2011;122:1405-10.

19. Milanov I. F-wave for assessment of segmental motoneuron excitability. Electromyogr Clin Neurophysiol 1992;32:11-5.

20. Milanov I. Neurographic studies in hemiplegic patients. Funct Neurol 1995;10:77-82.

21. Nakazumi Y, Watanabe Y. F-wave elicited during vol- untary contraction as a monitor of upper motor neuron disorder. Electromyogr Clin Neurophysiol 1992;32: 631-5.

22. Dumitru D, Amato AA, Zwarts MJ. Electrodiagnostic medicine. 2nd ed. Philadelphia: Hanley \& Belfus; 2002.

23. Chroni E, Katsoulas G, Argyriou AA, Sakellaropoulos GC, Polychronopoulos P, Nikiforidis G. Level of consciousness as a conditioning factor of $\mathrm{F}$ wave generation in stroke patients. Clin Neurophysiol 2006;117: 315-9.

24. Drozdowski W. The assessment of amplitude and occurrence of F waves in hemiparetic patients. Neurol Neurochir Pol 1993;27:479-91.

25. Drozdowski W, Kochanowicz J, Baniukiewicz E. F wave occurrence in patients after stroke. Neurol Neurochir Pol 1998;32:1341-52.

26. Choi IS, Kim JH, Han JY, Lee SG. The correlation between F-wave motor unit number estimation (FMUNE) and functional recovery in stroke patients. J Korean Med Sci 2007;22:1002-6.

27. Hara Y, Akaboshi K, Masakado Y, Chino N. Physiologic decrease of single thenar motor units in the F-response in stroke patients. Arch Phys Med Rehabil 2000;81:418-23. 